\title{
INFLUÊNCIA DAS FORÇAS DE INTERAÇÃO LÍQUIDO/GÁS NA ANÁLISE VIA CFD DO REATOR RH *
}

\author{
Johne Jesus Mol Peixoto ${ }^{1}$ \\ Weslei Viana Gabriel ${ }^{1}$ \\ Thiago Araújo Santos de Oliveira² \\ Natália Barros Barony ${ }^{3}$ \\ Carlos Antônio da Silva ${ }^{4}$ \\ Itavahn Alves da Silva ${ }^{5}$ \\ Varadarajan Seshadri ${ }^{6}$
}

\section{Resumo}

No modelamento matemático do reator $\mathrm{RH}$, a penetração do gás no líquido na perna de subida é o fator chave para determinar a distribuição do gás injetado e obter uma previsão confiável da taxa de circulação do líquido entre a câmara de vácuo e a panela. Neste trabalho, através de simulações numéricas via CFX, investigou-se o efeito das forças de arrasto, de sustentação, de lubrificação da parede, dispersão turbulenta e de massa virtual. A distribuição do gás na perna de subida e os valores de taxa de circulação previstos via CFX foram validados com resultados de modelo físico. A melhor predição da penetração do gás e da taxa de circulação foi obtida na simulação com incorporação da força de massa virtual e da força de sustentação, cujos coeficientes foram iguais a 0,3 e 0,1, respectivamente. A força de lubrificação deve ser utilizada para evitar o acúmulo de gás próximo às paredes da perna, mas possui efeito desprezível sobre a taxa de circulação. Os resultados dos modelos físico e matemático se ajustaram com a equação de Kuwabara para taxa de circulação.

Palavras-chave: CFD; Forças de não-arrasto; Modelamento Físico; Reator RH.

\section{CFD ANALYSIS OF INTER-PHASE FORCES IN LIQUID-GAS FLOW IN A RH REACTOR}

\section{Abstract}

Evaluation of gas penetration in the $\mathrm{RH}$ up leg is of primary importance as far as mathematical modeling of gas distribution and circulation features are regarded. In this study CFX has been used in order to assess the effects of drag force, lift force, wall lubrication force and turbulent dispersion force on gas penetration and circulation rate. The predicted radial gas distribution and liquid circulation have been validated against experimental data of physical model. The simulation results with incorporation of the virtual mass force coefficient of 0.3 and the lift force coefficient of 0.1 showed better predictions of gas distribution at the up-leg and circulation rate. In order to avoid the gas concentration near the up-leg walls, the wall lubrication force needs to be incorporated in the simulation, but it has a negligible effect on the circulation rate. Both experimental and CFD values fit to the Kuwabara equation for circulation rate.

Keywords: CFD; Non-drag Forces; Physical Modelling; RH Degasser.

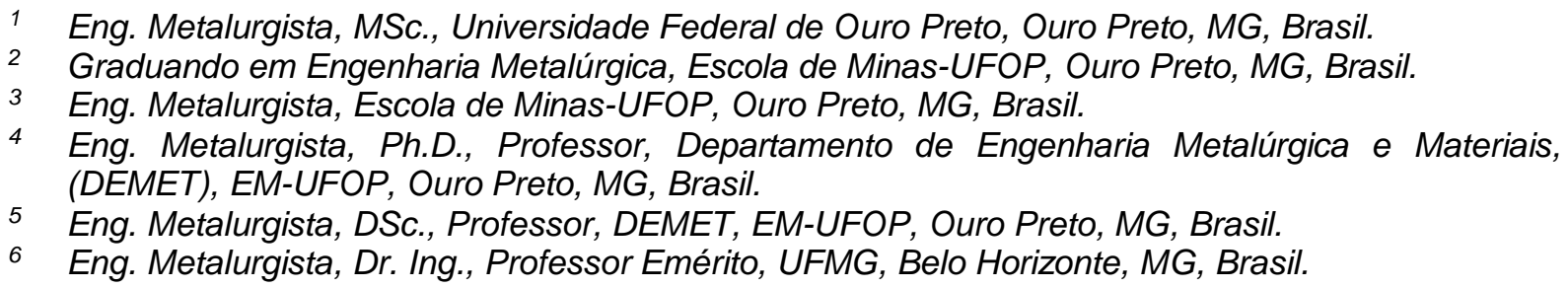




\section{INTRODUÇÃO}

O emprego de modelos físicos e matemáticos é recorrente quando se trata da análise da influência de parâmetros operacionais sobre o processo de desgaseificação no reator Rheinsahl-Heraeus $(\mathrm{RH})$. No tocante às simulações matemáticas, existem diferentes modelos de interação entre duas fases envolvidas e tipos de forças de nãoarrasto que podem ser empregados para prever a interação gás/líquido. A força de arrasto sempre existe quando uma bolha de gás se move em relação ao líquido, e geralmente, é a contribuição dominante para as forças de interação, desempenhando um papel essencial na determinação do fluxo multifásico (Chen et al., [1]). No entanto, a influência de forças de não-arrasto (força de sustentação, de massa virtual, de dispersão turbulenta e de lubrificação das paredes) pode também ser importante para descrever com maior fidelidade as características do escoamento multifásico. Além disso, Braga et al. [2] lembram que diversos modelos matemáticos não conseguiram boa representação da penetração de gás na perna de subida do reator $\mathrm{RH}$, e que, em algumas previsões, o gás permanece aderido às paredes da perna de subida. E de acordo com Geng et al. [3], a profundidade de penetração é o fator chave para determinar a distribuição do gás injetado, sendo que a saturação em fase gasosa no centro da perna de subida resulta na estabilização da taxa de circulação a partir de certa vazão.

Neves [4] avaliou diferentes parâmetros para as forças de não-arrasto e sua influência sobre a previsão da taxa de circulação e a fração volumétrica de gás na perna de subida do reator RH. Suas análises mostraram que para validar o modelo matemático foi necessário utilizar diferentes combinações de força de não-arrasto, sendo o coeficiente da força de sustentação igual 0,1 e os coeficientes da força de lubrificação $\mathrm{C} 1=-0,010$ e C2 $=0,050$, enquanto o coeficiente da força de dispersão turbulenta variou em função da vazão de gás. Desta forma, o autor notou uma leve melhoria na qualidade de previsão da penetração e uma maior dispersão da fase gasosa no interior da perna de subida, na região de encontro da pluma. No entanto, os resultados do modelo físico não foram completamente representados pela simulação, e o autor destaca ao final a necessidade de novas análises.

Neste sentido, Braga et al. [2] sugerem que o problema de penetração de gás identificado na literatura se deve a erros numéricos relacionados à discretização insatisfatórias das equações que governam o escoamento no desgaseificador $\mathrm{RH}$. Utilizando modelos físico e matemático com apenas um ou dois bicos injetores de gás, para contornar tal problema, propuseram um modelo matemático em regime transiente, com uma malha substancialmente mais refinada e esquema de advecção de $2^{\mathrm{a}}$ ordem para discretização do tempo. Obtiveram consideráveis melhorias na previsão da penetração de gás em comparação com os resultados do modelo físico. Porém, para a vazão de $5 \mathrm{~L} / \mathrm{min}$ por bico a dispersão de gás na perna foi subestimada e para as condições com dois bicos injetores com vazões maiores que $30 \mathrm{~L} / \mathrm{min}$, notouse uma tendência das bolhas do gás se concentrarem no centro da perna no modelo matemático, enquanto que o experimento físico indica a dispersão do gás por toda a perna.

Chen et al. [1] utilizando aproximação Euleriana-Euleriana avaliariam a influência de quatro forças de interação entre as fases gasosa e líquida sobre a taxa de circulação no reator $\mathrm{RH}$, em ambos, o modelo a frio (água/ar) e o reator real (aço/argônio). Encontraram que as forças de arrasto e de dispersão turbulenta apresentam enorme 
influência no fluxo multifásico, enquanto as forças de sustentação e de massa virtual possuem influência negligenciável.

Estes fatos atentam para a necessidade de otimização da previsão da penetração do gás no fluxo de aço na perna de subida para melhor representação do fluxo circulatório do aço entre a câmara de vácuo e a panela de modo a aumentar a confiabilidade dos modelos. Neste trabalho, investigou-se o efeito da combinação de forças de arrasto e forças de não-arrasto para previsão da penetração do gás na perna de subida e da taxa de circulação no modelo físico do reator $\mathrm{RH}$, em função da vazão de gás nos bicos injetores.

\section{MATERIAIS E MÉTODOS}

Dados geométricos do reator industrial e do modelo são mostrados na Tabela 1. 0 modelo do sistema RH foi construído em acrílico, obedecendo a um fator de escala $\lambda$ $=1: 7,5$. O adimensional de Froude $(\mathrm{Fr})$ foi utilizado como critério de similaridade entre o modelo e o protótipo, exceto os bicos de injeção, cujo diâmetro foi determinado a partir do número de Froude modificado $\left(\mathrm{Fr}_{\mathrm{m}}\right)$ :

$$
F r=\frac{V^{2}}{g D} ; \quad F r_{m}=\frac{u^{2} \rho_{g}}{D g \rho_{L}}
$$

Onde: $v$ - velocidade do líquido $(\mathrm{m} / \mathrm{s}) ; \rho_{g}$ - densidade do gás $\left(\mathrm{kg} / \mathrm{m}^{3}\right) ; \rho_{L}$ - densidade do líquido $\left(\mathrm{kg} / \mathrm{m}^{3}\right)$; $\mathrm{D}$ - diâmetro interno da perna $(\mathrm{m}) ; \mathrm{g}$ - aceleração da gravidade; $\mathrm{u}$ - Velocidade do Gás no Bico;

Tabela 1. Descrição das Características do Protótipo e do Modelo

\begin{tabular}{|c|c|c|c|}
\hline \multicolumn{2}{|c|}{ Caraterísticas Do Reator } & Protótipo & Modelo \\
\hline \multirow{3}{*}{$\begin{array}{c}\text { Dados } \\
\text { Operacionais }\end{array}$} & Capacidade de Vácuo (mBar) & 0,67 & 984 \\
\hline & Temperatura de Trabalho $\left({ }^{\circ} \mathrm{C}\right)$ & 1560 & 25 \\
\hline & Fluido & Aço & Água \\
\hline \multirow{2}{*}{ Vaso Superior } & Diâmetro Interno (mm) & 2245 & 299,33 \\
\hline & Altura $(\mathrm{mm})$ & 7600 & $70^{*}$ \\
\hline \multirow{4}{*}{$\begin{array}{l}\text { Vaso inferior } \\
\text { (Panela) }\end{array}$} & Di superior $(\mathrm{mm})$ & 3400 & 490 \\
\hline & Di inferior $(\mathrm{mm})$ & 3000 & 460 \\
\hline & Altura (mm) & 4250 & 566 \\
\hline & Altura líquido (mm) & 3650 & 486 \\
\hline \multirow{4}{*}{$\begin{array}{l}\text { Snorkels } \\
\text { (pernas) }\end{array}$} & Comprimento (mm) & 1820 & 242,67 \\
\hline & Diâmetro Interno (mm) & 750 & 100 \\
\hline & Distância entre Linhas de Centro (mm) & 1540 & 205,33 \\
\hline & Profundidade de Imersão $(\mathrm{mm})$ & 450 & 60 \\
\hline \multirow{6}{*}{$\begin{array}{l}\text { Sistema de } \\
\text { Injeção de Gás } \\
\text { de Arrasto }\end{array}$} & Gás & Argônio & Ar comprimido \\
\hline & Vazão (NL/min) & 2500 & 120 \\
\hline & Densidade de Gás $\left(\mathrm{Kg} / \mathrm{m}^{3}\right)$ & 0,13 & 1,123 \\
\hline & $\begin{array}{c}\text { Injeção de gás (Snorkel de } \\
\text { Subida) }\end{array}$ & $\begin{array}{l}6 \text { bicos, distribuídos em } 2 \\
\text { níveis de injeção }\end{array}$ & $\begin{array}{l}16 \text { bicos, } 2 \\
\text { níveis }\end{array}$ \\
\hline & Distância entre Níveis de Injeção (mm & 175 & 23,3 \\
\hline & Diâmetro do Bico de Injeção (mm) & 8 & 2,4 \\
\hline
\end{tabular}

*Altura necessária somente para elevação da água, devido o vácuo induzido.

\subsection{Modelo físico}

No modelamento físico do reator $\mathrm{RH}$, uma técnica usualmente empregada para determinar a taxa de circulação é o Método da Condutivimetria, como nos trabalhos 
de Seshadri e Costa [5], Vargas [6], Kishan e Dash [7], Neves [4], entre outros. Seguindo estes autores a técnica utilizada consistiu na injeção de um pulso salino ( $5 \mathrm{~mL}$ de solução aquosa saturada de $\mathrm{KCl}$ ), no interior da câmara de vácuo, próximo a perna de subida, enquanto o condutivímetro (um sensor de condutividade) posicionado no centro da perna de descida monitora continuamente a variação da concentração do sal na perna de descida, pela medição da variação de condutividade da água. O condutivímetro é conectado a uma placa de aquisição de dados, que por sua vez faz interface com um programa de computador, onde os dados de cada ensaio são armazenados e processados e, por conseguinte, a determinação da taxa de circulação através da Equação 2, obtida por meio de balanço de massa de traçador, como descrito no trabalho de Vargas [6]. Foram realizados cerca de 8 testes para cada condição experimental (vazão de gás). Os valores de taxa de circulação obtidos por esta técnica foram utilizados para validar o modelo matemático.

$$
Q=\Delta \mathrm{C} * \mathrm{~W} / A_{\text {pico }}
$$

Onde: $\mathrm{Q}$ - taxa de circulação em $\mathrm{Kg} / \mathrm{s} ; \Delta \mathrm{C}$ - o incremento de concentração de traçador em $\mathrm{g}$ de $\mathrm{KCl} / \mathrm{kg}$ de água; $\mathrm{W}$ - quantidade de líquido no reator em $\mathrm{kg}$; $A_{\text {pico }}$ - área da região correspondente à passagem do primeiro pulso de traçador sob a curva concentração versus tempo.

Para caracterizar a zona de injeção de gás, isto é, determinar a profundidade do bolsão de gás proveniente dos bicos injetores, foram realizadas filmagens da parte inferior da seção transversal da perna de subida, utilizando uma câmera de alta velocidade modelo PiXELINK PL- B776U, com software próprio para a aquisição de imagens. Análise de imagens foi realizada com o software AOS Imagem Studio. As imagens de penetração do bolsão de gás foram comparadas com resultados das simulações matemáticas.

\section{$2.2 \quad$ Modelo matemático}

A geometria utilizada nas simulações foi construída através do software Design Modeler do Ansys e suas dimensões seguem as dimensões do modelo em acrílico (Tabela 1). Pela condição de simetria, apenas metade do reator foi considerado. A malha foi construída utilizando o software Meshing Modeler, com elementos predominantemente tetraédricos. Utiliza-se a função avançada PARA proximidade e curvatura; aplica-se a ferramenta inflation em todas as superfícies externas (paredes) do reator, com cinco camadas e o parâmetro taxa de transição igual a 0,2. O estudo de independência de malha foi realizado através da comparação dos resultados de taxa de circulação obtidos com malhas de tamanhos variados. Obteve-se uma malha de $15 \mathrm{~mm}$ no vaso inferior utilizando a ferramenta element sizing, com refinamento na perna de subida de $2 \mathrm{~mm}$ e no restante da câmara de vácuo e na perna de descida de $5 \mathrm{~mm}$, totalizando cerca de 1,2 milhões de elementos e 370 mil nós (vide Figura 1.a).

As simulações matemáticas foram realizadas utilizando-se o software CFX 17.1 (Ansys $\AA$ ) versão acadêmica. $O$ modelo matemático considera o escoamento tridimensional e turbulento; os fluidos newtonianos, incompressíveis (desconsiderouse a expansão do gás), sistema isotérmico (a 25으), pressão ambiente igual a 1atm. Utilizou-se os valores padrões do software para as propriedades físicas da água e do ar $\left(\mathrm{a} 25^{\circ} \mathrm{C}\right)$. $\mathrm{O}$ modelo de turbulência adotado é o modelo $\mathrm{k}-\varepsilon$ para a fase contínua 
(líquido, emulador do aço líquido), enquanto para a fase discreta (ar, emulador do argônio), adota-se o modelo de equação zero de fase dispersa, que assume que a fase discreta possui a mesma viscosidade cinemática turbulenta da fase contínua [8].

Utiliza-se o modelo de diferença de densidades como modelo de flutuação, o modelo de partículas para interfaces, sendo que a transferência de turbulência entre as fases é estimada pelo modelo de Sato [8]. No CFX, as equações diferenciais de escoamento são resolvidas através do método de volumes finitos, e adota-se a abordagem multifásica euleriana-euleriana, na qual são resolvidas as seguintes equações:

- Equação de conservação de massa de cada fase (água e ar);

$$
\frac{\partial}{\partial t}\left(\alpha_{i} \rho_{i}\right)+\nabla \cdot\left(\alpha_{i} \rho_{i} \vec{u}_{i}\right)=0
$$

Onde: $\alpha_{i}, \rho_{i}$ e $u_{i}$ são, respectivamente, fração volumétrica, densidade e velocidade da fase líquida $(\mathrm{i}=\mathrm{l})$ e gasosa $(\mathrm{i}=\mathrm{g})$;

- Equação de conservação de volume, isto é, soma das frações volumétricas de ar e água igual a 1;

- Equações de conservação de energia cinética turbulenta e da taxa de dissipação da energia cinética de turbulência (modelo k- $\varepsilon$ ).

- Equações de conservação de quantidade de movimento de cada fase, água e ar (forma turbulenta das equações de Navier-Stokes), nas três direções cartesianas ( $x, y$ e $z)$ :

$\frac{\partial}{\partial t}\left(\alpha_{i} \rho_{i} \vec{u}_{i}\right)+\nabla \cdot\left(\alpha_{i} \rho_{i} \vec{u}_{i} \vec{u}_{i}\right)=-\alpha_{i} \nabla \mathrm{p}+\nabla \cdot\left[\alpha_{i}\left(\mu_{l}+\mu_{l, t}\right)\left(\nabla \vec{u}_{i}+\left(\nabla \vec{u}_{i}\right)^{T}\right)\right]+\alpha_{i} \rho_{i} \vec{g}+\vec{F}_{i}$

Onde: $\mathrm{g}$ - aceleração da gravidade, $\mu_{l}$ - viscosidade do líquido, $\mu_{l, t}$ - viscosidade turbulenta do líquido, determinada pelo modelo de turbulência k- $\varepsilon$, p - pressão, e $\vec{F}_{i}$ - força de interação entre as duas fases:

$$
\vec{F}_{l}=-\vec{F}_{g}=\vec{F}_{\text {Drag }}+\vec{F}_{\text {lift }}+\vec{F}_{V M}+\vec{F}_{T D}+\vec{F}_{W L}
$$

Onde: $\vec{F}_{\text {Drag }}$ - força de arrasto; $\vec{F}_{\text {lift }}$ - força de sustentação; $\vec{F}_{V M}$ - força de massa virtual e $\vec{F}_{T D}$ - força de dispersão turbulenta; $\vec{F}_{W L}$ - força de lubrificação da parede.

Força de arrasto: Para o fluxo multifásico em agitação gasosa no reator $\mathrm{RH}$, o arrasto tenderá a diminuir a velocidade da bolha e acelerará o líquido envolvente devido à dissipação viscosa (Eq. 6):

$$
\vec{F}_{\text {Drag }}=C_{D} \frac{3 \alpha_{g} \alpha_{l} \rho_{l}}{4 d_{g}}\left|\vec{u}_{g}-\vec{u}_{l}\right|\left(\vec{u}_{g}-\vec{u}_{l}\right)
$$

Onde: $d_{g}$ - diâmetro da bolha de gás; $C_{D}$ é o coeficiente de arrasto, o qual pode ser estimado pelo modelo de Grace, modelo de Ishii-Zuber, ou pode-se utilizar um valor constante, como por exemplo, $\mathrm{C}_{\mathrm{D}}=0,44$.

O modelo de Grace foi desenvolvido utilizando dados da interação água-ar para fluxos com distribuição esparsa de bolhas, e no caso de elevada fração volumétrica de gás, deve-se utilizar o coeficiente de correção de fração volumétrica, o qual depende do tamanho das bolhas, sendo igual a 2 para bolhas relativamente grandes. O modelo de Ishii Zuber para a força de arrasto é apropriado para fluxos contendo elevada 
concentração de partículas, e no CFX, este modelo determina automaticamente o valor de $C_{D}$ em função dos números adimensionais de Reynolds e de Eotvos - razão entre força gravitacional e de tensão superficial [8].

Força de sustentação: causada principalmente pela existência de um gradiente de velocidade no campo de fluxo do líquido, o qual é perpendicular à direção do fluxo principal, atuando sobre a bolha de gás (Chen et al. [1]):

$$
\vec{F}_{\text {Lift }}=-C_{L} \rho_{l} \alpha_{g}\left(\vec{u}_{g}-\vec{u}_{l}\right) \times\left(\nabla \times \vec{u}_{l}\right)
$$

$C_{L}$ é o coeficiente de sustentação, que para o fluxo viscoso, varia de 0,01 a 0,5 de uma forma que é apenas parcialmente compreendida [8].

Força de massa virtual (VMF): também chamada de força de massa adicionada, 0 efeito de massa virtual (Eq. 8) pode ocorrer quando uma bolha acelera em relação ao líquido, sendo definida da seguinte forma (Chen et al. [1]):

$$
\vec{F}_{V M}=C_{V M F} \rho_{l} \alpha_{g}\left(\frac{d_{l} \vec{u}_{l}}{\partial t}-\frac{d_{g} \vec{u}_{g}}{\partial t}\right)
$$

$C_{V M F}$ é o coeficiente de massa virtual, e possui um valor de 0,5 para o fluxo invíscido em torno de uma esfera isolada. Em geral, depende da forma e da concentração das bolhas, e deve ser especificado [8].

Força de dispersão turbulenta (TDF): pode agir na bolha devido à flutuação de turbulência do líquido (Chen et al. [1]). O modelo utilizado neste trabalho foi o modelo baseado na média de Favre (ou média ponderada pela massa) da força de arrasto, mais indicado nas situações onde não se conhece um valor apropriado para o coeficiente de dispersão turbulenta, $C_{T D}[8]$ :

$$
\vec{F}_{T D}=-C_{T D} C_{c d} \frac{v_{l}}{\sigma_{l g}}\left(\frac{\nabla \alpha_{g}}{\alpha_{g}}-\frac{\nabla \alpha_{l}}{\alpha_{l}}\right)
$$

$C_{T D}$, valor padrão igual a $1 ; C_{c d}$ é o coeficiente de transferência de quantidade de movimento para a força de arrasto; $v_{l}$ é o volume específico de líquido; $\sigma_{t c}$ é o número de Schmidt turbulento, usualmente igual a 0,9 [8].

Força de lubrificação da parede (WLF): em um tubo vertical, o fluxo de subida de bolhas tende a se concentrar numa região próxima à parede, mas não imediatamente adjacente à parede do tubo [8]. O modelo da força de lubrificação de Frank foi utilizado para modelar este efeito, que tende a afastar a fase dispersa (ar) para longe das paredes:

$$
F_{W L}=C_{W L} \rho_{l} \alpha_{g}\left|U_{l}-U_{g}\right|^{2} n_{w}
$$

$C_{W L}$, é o coeficiente da força de lubrificação, dado pelo modelo de Frank; nw = distância normal da parede; $U_{I}-U_{g}=$ diferença de velocidade relativa entre as fases, em um plano normal a $n_{w}$ próximo a parede. Ressalta-se que esta força atua apenas em uma camada adjacente à parede, até certa distância dada pelo modelo adotado e que é função do diâmetro das bolhas [8]. 


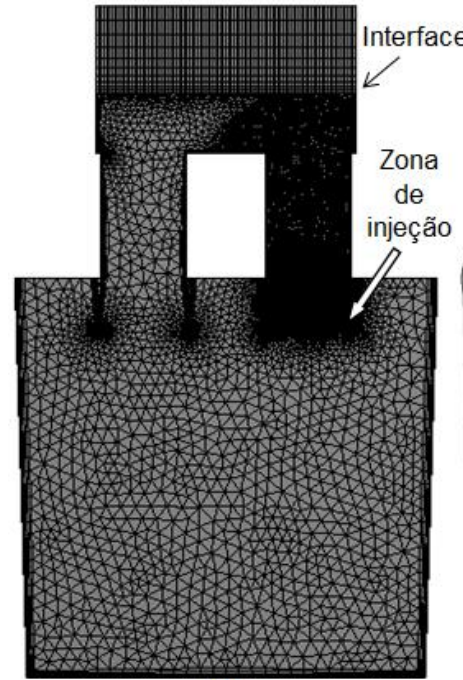

(a)

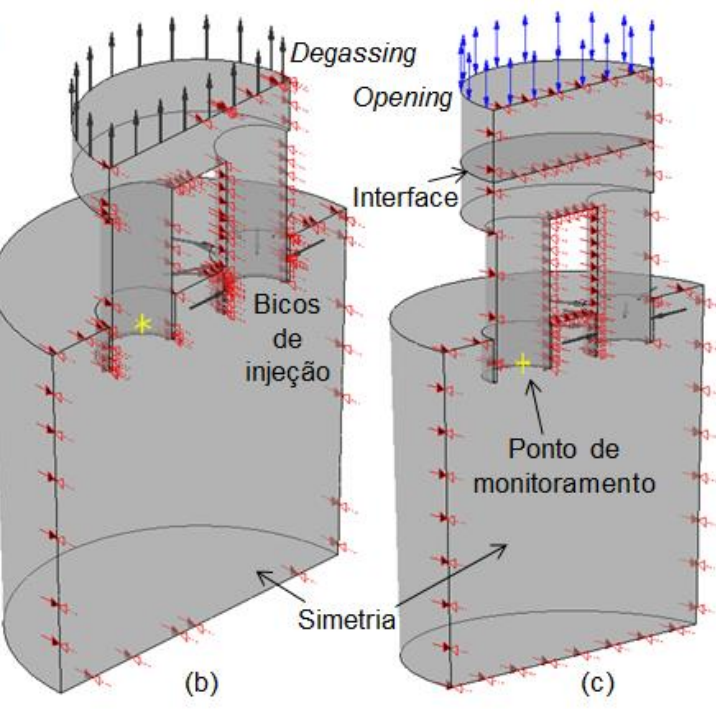

(b)

(c)

Figura 1. a) Malha construída para o modelo com camada de ar na câmara de vácuo; Vista em perspectiva da geometria do reator $\mathrm{RH}$, destacando as principais partes do setup para o modelo: $\mathrm{b}$ ) sem camada de ar e c) camada de ar de $10 \mathrm{~cm}$.

Os modelos matemáticos existentes no CFX para as forças de arrasto e de não arrasto foram testados de modo a obter uma combinação entre estas forças que resulte em maior equivalência com resultados dos testes no modelo físico. As condições de contorno aplicáveis ao problema são (vide Figura 1.b e 1.c):

- Condição de não deslizamento, aplicada em todas as paredes, regiões onde o fluido possui velocidade zero;

- Condição de simetria, o que implica que o cálculo em questão abrange apenas metade de toda a geometria definido um plano de simetria e nessa região as componentes de velocidade normais à fronteira são iguais a zero.

- Condição de entrada: bicos injetores, onde a injeção de gás é configurada considerando-se a condição de simetria de forma a simular vazões de $30,40,50$, $60,70,80,90,100,110$ e 120L/min convertidos para valores de vazão mássica $(\mathrm{kg} / \mathrm{s})$. O regime de escoamento selecionado é o subsônico, com uma intensidade de turbulência de $5 \%$ (média).

- Condição de escorregamento livre na superfície da panela;

- Superfície da câmara de vácuo: sem camada de ar - condição de desgaseificação, que permite saída apenas do gás; com camada de ar de $10 \mathrm{~cm}$ - condição de abertura, com pressão igual ao vácuo aplicado.

Para simplificação do modelo, despreza-se a deformação das bolhas de gás, assim como a interação entre bolhas (quebra e coalescência). Deste modo, assume-se que o diâmetro da bolha de gás é constante, sendo calculado para cada vazão a partir da correlação (Eq. 11) experimental reportada por Sano et al. [9]:

$$
d_{g}=0,091\left(\frac{\sigma_{l}}{\rho_{l}}\right)^{0,5} u_{g, 0}^{0,44}
$$

Onde: $u_{g, 0}$ - velocidade do gás na saída do bico de injeção $(\mathrm{m} / \mathrm{s}) ; \sigma_{l}$ tensão interfacial líquido/gás ( $\mathrm{N} / \mathrm{m})$;

Apesar do elevado caráter transiente do fluxo de aço líquido no interior do reator $\mathrm{RH}$, alguns parâmetros podem ser avaliados com boa precisão a partir de simulações 
matemáticas em regime permanente, economizando considerável tempo computacional. Para solução das equações diferenciais do modelo computacional, foi utilizado esquema de advecção de $1^{\text {a }}$ ordem (Upwind) com alta resolução (High Resolution no ANSYS CFX) para todas as equações de conservação e do modelo de turbulência. Devido à oscilação de alguns parâmetros, foi utilizado o controle de escala de tempo física igual a 0,005s e um máximo de 2500 iterações.

\section{RESULTADOS E DISCUSSÃO}

\subsection{Penetração do gás no líquido na perna de subida}

Modelos físicos do Reator $\mathrm{RH}$ permitem a caracterização da zona de injeção ou pluma de gás na perna de subida, parte fundamental para comparação e validação do modelo matemático, como realizado em trabalhos anteriores (Neves [4]; Silveira [10]). Neste sentido, na Figura 2 nota-se que a penetração do gás logo na saída dos bicos é pequena (cerca de $1 \mathrm{~cm}$ ), e a medida que sobe pela perna, a penetração do gás aumenta, atingindo o centro da perna antes de entrar na câmara de vácuo. A Figura 3 apresenta imagens da zona de injeção obtidas por simulação matemática para diferentes combinações das forças de arrasto e de não-arrasto. A força de arrasto foi considerada em todos os casos, devido a sua importância no fluxo viscoso. Estas imagens foram obtidas através de dois planos transversais à perna de subida passando nos dois níveis de injeção, sendo que no plano do nível inferior foi utilizado transparência de $50 \%$ para permitir a visualização simultânea dos dois níveis de injeção.

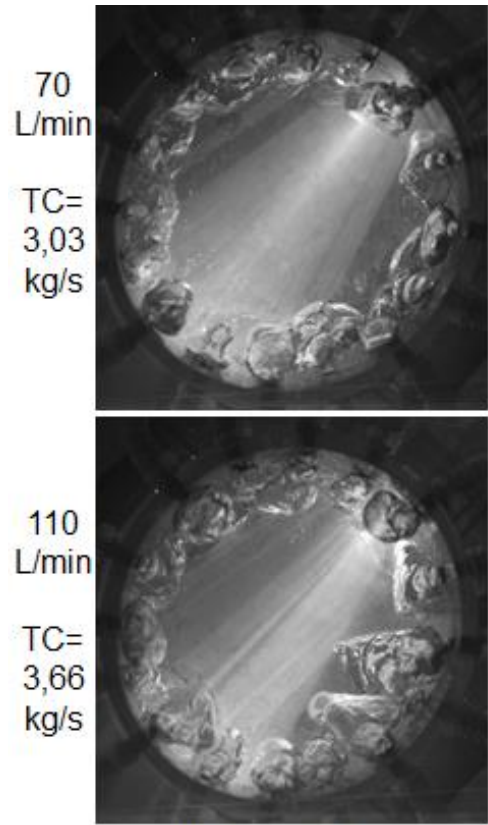

a) Região dos bicos

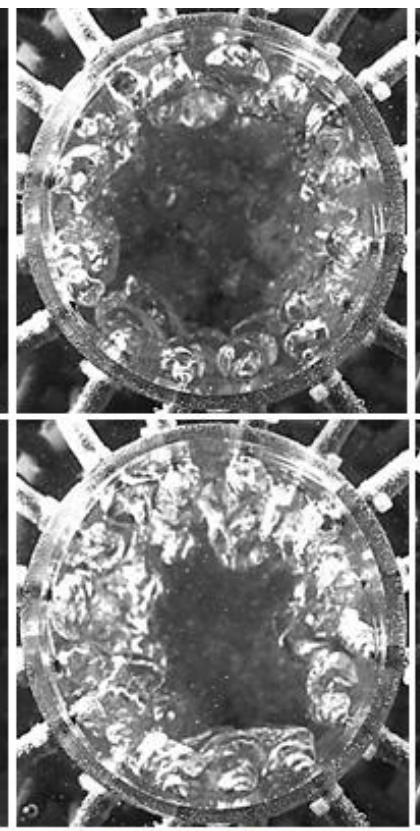

b) Foco inferior

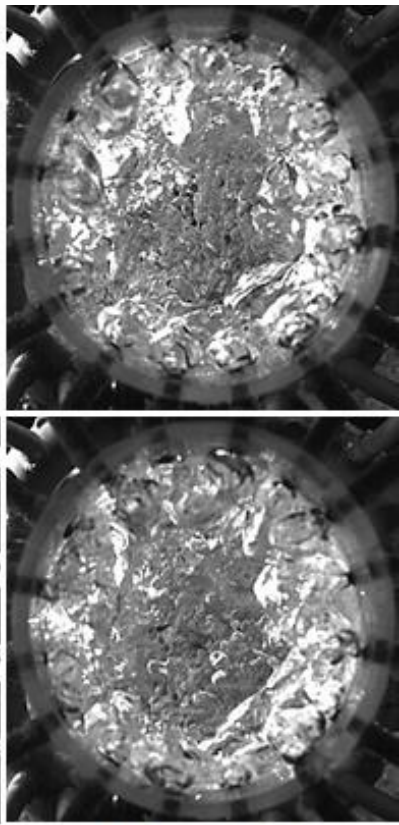

c) Foco superior

Figura 2. Penetração do gás na zona de injeção do modelo físico, imersão das pernas do reator $\mathrm{RH}$ : $60 \mathrm{~mm}$, vazão de gás $70 \mathrm{l} / \mathrm{min}$ (superior) e 110l/min (inferior), com o foco da câmera na região: (a) dos bicos de injeção; (b) inferior da perna e (c) superior da perna TC - taxa de circulação.

Para a vazão de gás igual a 110L/min, a taxa de circulação obtida no modelo físico foi $3,66 \mathrm{~kg} / \mathrm{s}$. Pela Figura 3.a, quando se utiliza apenas a força de arrasto, qualquer dos três modelos, a penetração do gás e a taxa de circulação de líquido são muito inferiores aos resultados do modelo físico. Neves [4] também não identificou aumento 
na penetração de gás do fluxo ascendente de líquido com introdução das forças sustentação e dispersão turbulenta. Nota-se que apenas a força de massa virtual (Figura 3.c) foi responsável pelo aumento efetivo da penetração do gás no fluxo de líquido, sendo superior a penetração observada no modelo físico (Figura 2.a). Isto é ajustável pelo coeficiente $C_{V M}$, uma vez que o valor 0,5 é indicado para o fluxo de bolhas esféricas isoladas. Esta análise é apresentada na Figura 4. O valor $\mathrm{C}_{\mathrm{VMF}}=0,3$ resultou na melhor representatividade da penetração do gás em comparação ao modelo físico, porém, o valor de taxa de circulação foi abaixo do valor real.

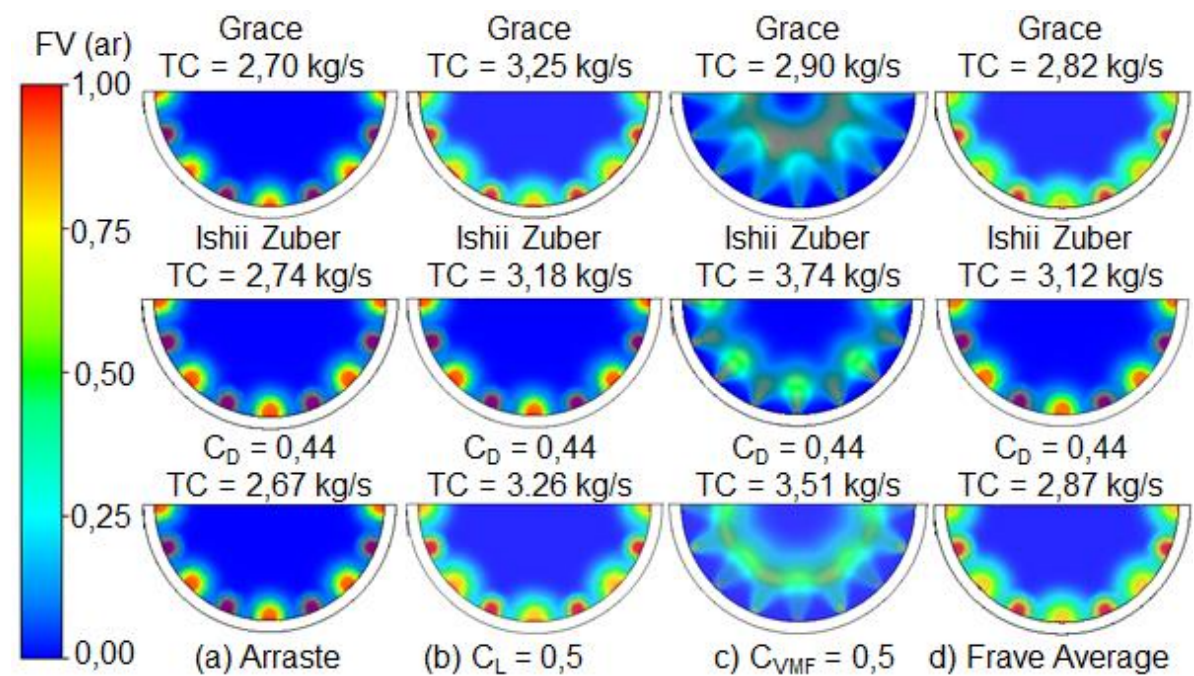

Figura 3. Penetração do gás na zona de injeção do modelo matemático, imersão das pernas do reator $\mathrm{RH}: 60 \mathrm{~mm}$, vazão de gás $110 \mathrm{l} / \mathrm{min}$. Influência das forças de interação gás/líquido, força de: (a) arrasto (apenas); (b) sustentação; (c) massa virtual; (d) dispersão turbulenta. FV - fração volumétrica de ar.

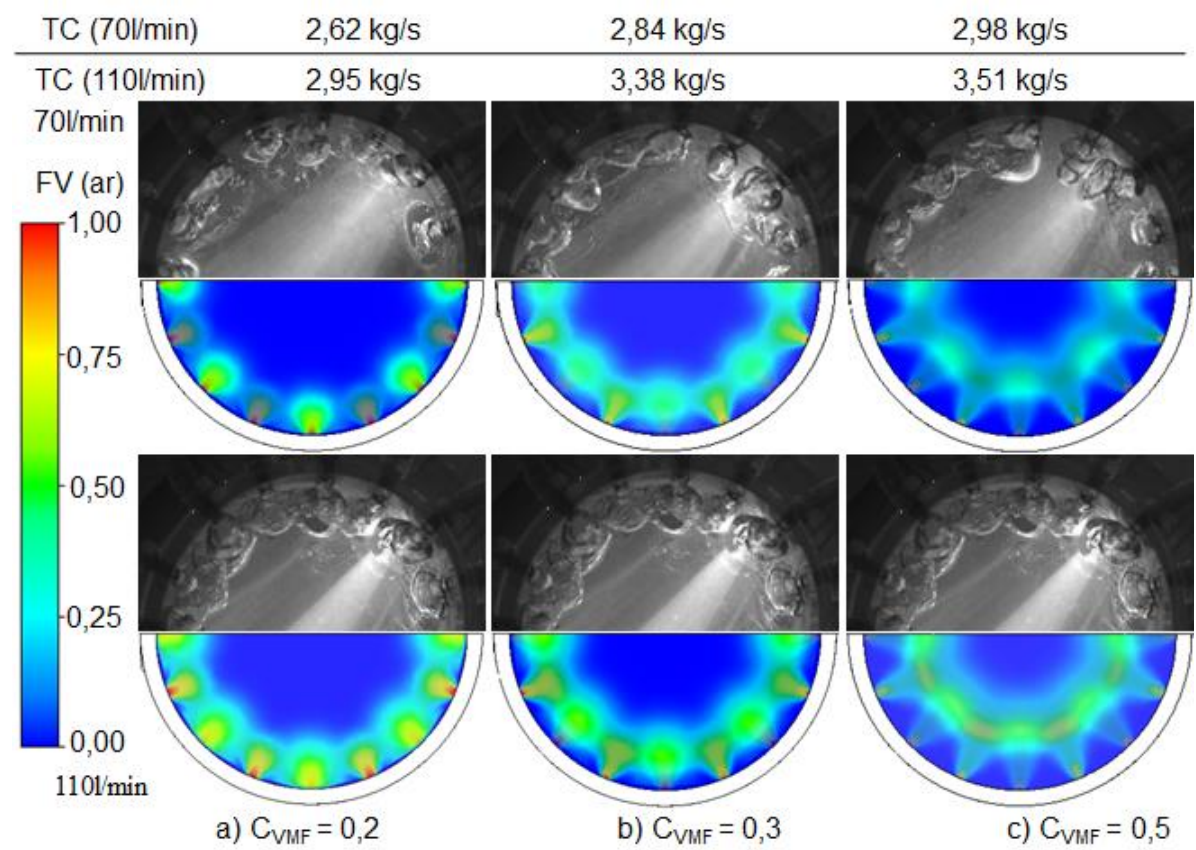

Figura 4. Profundidade de penetração do gás na zona de injeção, imersão das pernas $60 \mathrm{~mm}$, vazão de gás $70 \mathrm{l} / \mathrm{min}$ (superior) e 110l/min (inferior), modelo físico versus matemático utilizando $C_{D}=0,44 \mathrm{e}$ força de massa virtual: (a) $C_{V M F}=0,2$; (b) $C_{V M F}=0,3$; (c) $C_{V M F}=0,5$.

A força de massa virtual promoveu melhorias na previsão da penetração do gás na zona de injeção do gás inerte, mas apenas na região próxima aos bicos onde a 
aceleração relativa entre as fases é grande. Desta forma ao longo da perna de subida o gás permaneceu aderido à parede da perna, conforme Figura 5.a. Para solucionar tal problema, acrescenta-se ao modelo a força de lubrificação da parede, que remove o acúmulo de gás na parede (Figura 5.b), porém, com efeito insignificante sobre a taxa de circulação de líquido e sobre a distribuição do gás para o centro da perna de subida do $\mathrm{RH}$. As forças de sustentação e de dispersão turbulenta foram acrescentadas para melhorar a distribuição do gás na parte superior da perna de subida e elevar a taxa de circulação. Neste sentido, a força de sustentação apresentou melhor resultado aliado a maior elevação da superfície livre na região de saída do gás inerte no topo da perna de subida do $\mathrm{RH}$. Torna-se necessário ainda ajustar o valor de $\mathrm{C} L$, uma vez que ele depende fortemente do formato das bolhas e decresce com o diâmetro da bolha- $d_{b}$ (Zhang et al. [11]).

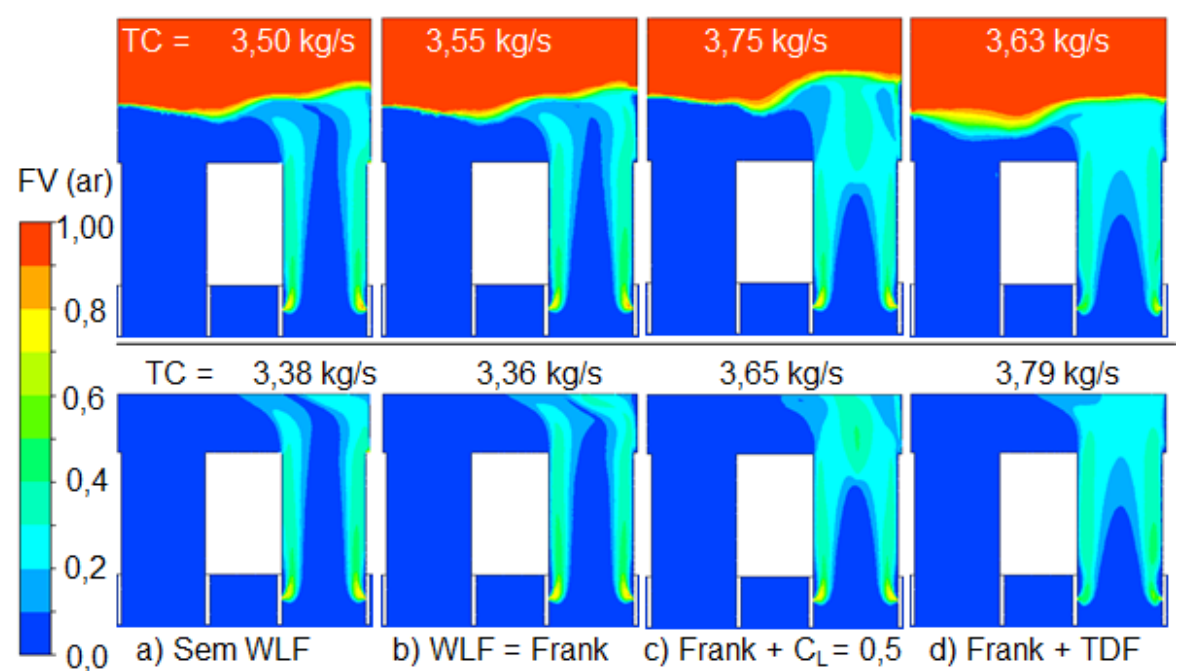

Figura 5. Influência das forças de não-arrasto sobre a penetração do gás no fluxo ascendente de líquido; combinação de $C_{D}=0,44, C_{V M F}=0,3$ e: (a) sem WLF; (b) WLF = modelo de Frank; (c) WLF + $C_{L}=0,5$; (d) $W L F+T D F$. Imersão das pernas $60 \mathrm{~mm}$, vazão de gás $110 \mathrm{l} / \mathrm{min}$.

Os resultados descritos até aqui indicam que o problema de penetração de ar encontrado na literatura está relacionado com a atuação da força de massa virtual, como sugerido por Geng et al. [3] e observado em outros trabalhos recentes que utilizaram $\mathrm{C}_{\mathrm{Vm}}=0,5$ (sobre $\mathrm{RH}$ - Kishan e Dash [7] e Ling et al. [12]; em outros casos - Zhang et al [11]). No entanto, não existe consenso sobre a atuação da força de massa virtual, visto que outros autores atestam que seu efeito é negligenciável (Chen et al. [1], Díaz et al. [13]).

\subsection{Taxa de Circulação}

A Figura 6.a apresenta resultados de taxa de circulação de líquido para diferentes valores do coeficiente da força de sustentação $\left(C_{L}\right)$, além do resultado para o modelo com superfície fixa (sem camada de ar na câmara de vácuo). Os resultados do modelo matemático considerado se aproximam mais dos dados experimentais quando $\mathrm{C}_{\mathrm{L}}=$ 0,1 . Este valor foi adotado anteriormente no trabalho de Ling et al. [12]. O modelo com superfície fixa na câmara de vácuo (Figura 1.b) também apresenta valores coerentes com os resultados experimentais, mas adotando $\mathrm{C}_{\mathrm{L}}=0,5$.

Kuwabara et al. [14] propuseram uma relação empírica para determinação da taxa de circulação $\left(\mathrm{Q}_{\mathrm{c}}\right)$ no reator $\mathrm{RH}(\mathrm{Eq} .12)$ : 


$$
Q c \propto Q_{g}^{\frac{1}{3}} D_{l}^{\frac{4}{3}}\left[\ln \left(\frac{P_{b}}{P_{C V}}\right)\right]^{\frac{1}{3}}
$$

Onde $Q_{g}$ : vazão de gás ( $\left.\mathrm{Nm}^{3} / \mathrm{s}\right)$; D:: Diâmetro da perna $(\mathrm{m})$; $\mathrm{P}_{\mathrm{b}}$ e $\mathrm{P}_{\mathrm{cv}}$, pressão na ponta dos bicos de injeção e na câmara de vácuo, respectivamente.

A relação entre taxa de circulação (TC) e $\mathrm{Qg}^{1 / 3} \mathrm{D}_{\mathrm{leg}}{ }^{4 / 3}$ é apresentada na Figura 6.b. Nota-se boa concordância entre os resultados obtidos pela técnica da condutivimetria e os valores calculados via CFD, os coeficientes angulares das duas curvas foram muito próximos, indicando que o modelo matemático é capaz de prever os valores da taxa de circulação de líquido entre a câmara de vácuo e a perna de subida.
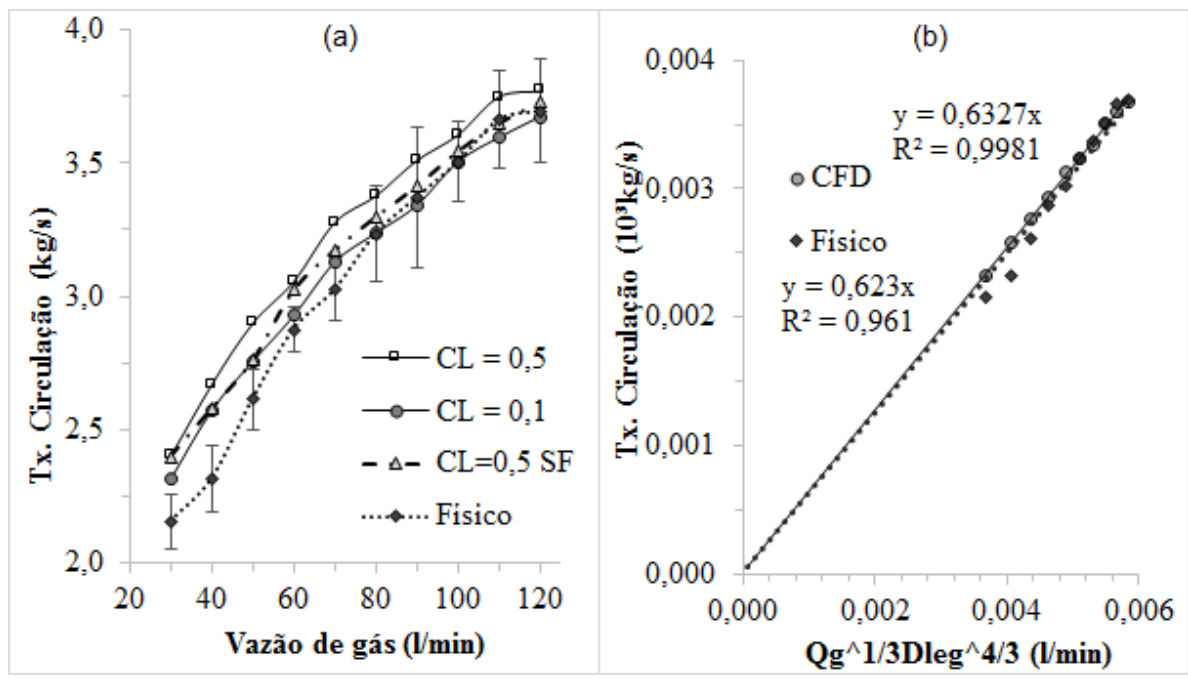

Figura 6. (a) Taxa de circulação obtida no modelo físico e no modelo CFD com $C_{D}=0,44, C_{V M F}=0,3$, $\mathrm{WLF}=$ Frank e diferentes valores de $\mathrm{C} \mathrm{L}$; (b) Relação entre taxa de circulação de líquido e $\left(\mathrm{Q}_{\mathrm{g}}{ }^{1 / 3} \mathrm{D}_{\text {leg }}{ }^{4 / 3}\right)$. ${ }^{*} \mathrm{SF}$ - modelo com superfície fixa (câmara de vácuo).

A utilização dos modelos para as forças de não-arrasto no modelamento do reator $\mathrm{RH}$ como proposto neste trabalho reduz consideravelmente os custos computacionais, sendo necessário apenas algumas horas para se obter resultados muito similares ao observado no modelo físico. Seu emprego apresenta grande vantagem frente à simulação em regime transiente como realizado em trabalhos anteriores (Braga et al. [2], Ling et al. [12]) e pode ser utilizado para se determinar resultados preliminares para o fluxo no interior do $\mathrm{RH}$ que serão utilizados como condição inicial de simulações em regime transiente, reduzindo o tempo computacional total para solução do problema.

\section{CONCLUSÃo}

Imagens da distribuição de gás na perna de subida e medidas da taxa de circulação obtidas no modelo físico foram utilizadas para validar o modelo matemático. Os resultados de simulação matemática com incorporação das forças de não-arrasto mostraram que:

- a força de massa virtual foi responsável pelo aumento da penetração do gás próxima a zona de injeção do gás, na perna de subida, sendo que os melhores resultados são obtidos utilizando-se o coeficiente (CVMF) igual a 0,3; 
- em simulações em regime permanente, a força de lubrificação das paredes deve ser utilizada para evitar o acúmulo de gás próximo às paredes da perna de subida;

- a força de sustentação é importante para aumentar a penetração do gás no fluxo de líquido nas regiões superiores da perna;

- para o modelo com superfície livre, o valor do coeficiente $C_{L} 0,1$ forneceu resultados mais coerentes para a taxa de circulação;

- o efeito da força de dispersão turbulenta sobre a penetração do gás e sobre a taxa de circulação do líquido foi inferior às demais forças e, portanto foi negligenciada;

- os valores preditos para a taxa de circulação, via modelagem matemática, concordam com os resultados experimentais do modelo físico. Além disso, estes dados se ajustaram com a equação de Kuwabara [14] para cálculo da taxa de circulação.

\section{Agradecimentos}

Ao CNPq, à CAPES, à Fundação Gorceix e à FAPEMIG pelo apoio.

\section{REFERÊNCIAS}

1 Chen G, He S, Li Y, Guo Y, Wang Q. Investigation of Gas and Liquid Multiphase Flow in the Rheinsahl-Heraeus $(\mathrm{RH})$ Reactor by Using the Euler-Euler Approach. JOM. 2016; 68 (8): 2138-2148.

2 Braga BM, Neves L, Tavares RP. Simulação Física e Matemática da Penetração de Gás na Perna de Subida de um Modelo Físico de Desgaseificador RH. In: Associação Brasileira de Metalurgia, Materiais e Mineração. Anais do $47^{\circ}$ Seminário de Aciaria Internacional; 2016; Rio de Janeiro, Brasil. São Paulo: ABM; 2016. 354-363.

3 Geng D-Q, Lei H, He J-C. Numerical simulation of the multiphase flow in the RheinsahlHeraeus $(\mathrm{RH})$ system. Metallurgical and Materials Transactions B. 2010; 41(1), 234-247.

4 Neves L. Modelagem do escoamento multifásico no desgaseificador $\mathrm{RH}$ e no distribuidor de lingotamento contínuo [tese de doutorado]. Belo Horizonte: UFMG; 2012.

5 Seshadri V, Costa SLS. Cold model of RH degassing. Transactions of ISIJ. 1986; 26: 33138.

6 Vargas, GA. Modelagem Matemática e Física do Desgaseificador a Vácuo $\mathrm{RH}$ da Companhia Siderúrgica Tubarão - CST [dissertação de mestrado]. Belo Horizonte: UFMG; 2000.

7 Kishan PA, Dash SK. Prediction of Circulation Flow Rate in the RH Degasser Using Discrete Phase Particle Modeling. ISIJ International. 2009; 49 (4): 495-504.

8 ANSYS: ANSYS CFX- Theory Guide 17.1. Canonsburg: ANSYS; 2016.

9 Sano M, Mori K. Trans. ISIJ. 1980, 20: 675-81 (apud Geng et al. [3]).

10 Silveira WJ. Modelamento matemático do escoamento de fluidos no processo RH para previsão da taxa de circulação do aço [dissertação de mestrado]. Belo Horizonte: UFMG; 2011.

11 Zhang $\mathrm{Y}$, Bai $\mathrm{Y}$, Wang $\mathrm{H}$. CFD analysis of inter-phase forces in a bubble stirred vessel. Chemical Engineering Research and Design. 2013; 9 (I): 29-35.

12 Ling H, Li F, Zhang F, Conejo A N. Investigation on the Effect of Nozzle Number on the Recirculation Rate and Mixing Time in the RH Process Using VOF + DPM Model. Metallurgical and Materials Transactions B. 2016; 47B: 1950-1961.

13 Díaz ME, Iranzo A, Cuadra D, Barbero R, Montes FJ, Galán MA. Numerical simulation of the gas-liquid flow in a laboratory scale bubble column Influence of bubble size distribution and non-drag forces. Chemical Engineering Journal. 2008; 139: 363-379.

14 Kuwabara T, Umezawa K, Mori K, Watanabe H. Investigation of decarburization behavior in $\mathrm{RH}-$ Reactor an its operation improvement. Trans. ISIJ. 1981; 28: 305-314. 